\title{
Desarrollo y rendimiento de calabacín y lechuga cultivados sobre acolchados vivos en Cuenca, Ecuador
}

\section{Development and yield of zucchini and lettuce under living mulches in Cuenca, Ecuador}

\author{
Pedro Zea ${ }^{1}$, Laude Pierre ${ }^{1}$, Gabriela Lucero ${ }^{1}$, Walter Larriva ${ }^{1}$, Eduardo J. Chica ${ }^{1 *}$ \\ ${ }^{1}$ Facultad de Ciencias Agropecuarias, Universidad de Cuenca, Cuenca, Ecuador. \\ 凶 Autor para correspondencia: eduardo.chica@ucuenca.edu.ec
}

\begin{abstract}
Resumen
El mantenimiento de la cobertura del suelo a través de prácticas como los acolchados vivos ha sido reportado ampliamente como una de las prácticas más efectivas para reducir el riesgo de erosión, principalmente en zonas de pendiente. No obstante, su aplicación no siempre es compatible con los requerimientos agronómicos de muchos cultivos hortícolas. En este estudio evaluamos el efecto de dos sistemas de acolchado vivo en el desarrollo y rendimiento del calabacín y lechuga, cultivos hortícolas comúnmente cultivados en sistemas de producción periurbanos de Cuenca (Ecuador). Los experimentos se desarrollaron usando diseños de bloques completos al azar con 5 repeticiones y tres tratamientos: acolchado vivo de trébol, acolchado vivo de vegetación espontánea manejada y un control. Los resultados muestran que los cultivos sobre algunos acolchados pueden alcanzar rendimientos similares a los observados en el sistema de siembra tradicional sin cobertura. No obstante, la implementación y manejo de los sistemas de acolchado vivo requirieron mayores recursos económicos y trabajo que el sistema tradicional sin cobertura. Estos resultados ayudan a identificar posibles limitaciones en la implementación de sistemas de acolchado vivo en cultivos hortícolas que deberán ser satisfechas previo a su posible implementación comercial. Adicionalmente, estos resultados resaltan la importancia de conducir evaluaciones locales de este tipo de estrategias de conservación de suelos.
\end{abstract}

Palabras clave: cobertura de suelo, agricultura de conservación, horticultura, calabacín, lechuga.

\begin{abstract}
Keeping soils covered through living mulches is one of the most widely reported strategies to reduce the risk of soil erosion on sloped lands. Nevertheless, soil covers application is not always compatible with the agronomic requirements of vegetable crops. Here, we assess the effect of two living mulches on development and yield of zucchini and lettuce, these crops are typically cultivated in peri-urban systems around Cuenca (Ecuador). A randomized complete block design with 5 replications and three treatments (clover mulch, managed spontaneous natural growth mulch and control) was used. Results shown that yield crops on some mulches are similar yields of those uncovered traditional crops systems. However, implementing and maintaining these mulch crops required more economic resources and labor than uncover traditional crops. These result help identify potential limitations for the commercial implementation of living mulches in vegetable crops under Cuenca conditions and highlight the need to conducts local evaluations of soil conservation strategies.
\end{abstract}

Keywords: soil cover, conservation agriculture, horticulture, zucchini, lettuce. 


\section{Introducción}

El cultivo de hortalizas para consumo fresco en Ecuador se concentra mayoritariamente en la región interandina (FAO, 2005). Este tipo de producción es desarrollada por miles de pequeños y medianos productores principalmente en zonas cercanas a centros poblados. Debido a la topografía de la sierra ecuatoriana y la competencia con otros usos de suelo, las fincas hortícolas se ubican tanto en terrenos planos con buena aptitud agrícola como en terrenos con limitaciones productivas, caracterizados por pendientes pronunciadas y mayor susceptibilidad a la erosión. Por ejemplo, Harden (1988) estimó tasas de erosión anuales de entre 5 y $101 \mathrm{t} \cdot \mathrm{ha}^{-1}$ en zonas bajas de la cuenca del río Ambato donde los cultivos de hortalizas son comunes. Además, de los factores topográficos y ambientales, el riesgo de erosión en sistemas de producción de hortalizas se incrementa aún más debido al requerimiento frecuente de procesos de labranza y control de arvenses que reducen la cobertura vegetal del suelo y alteran su estructura.

La cobertura vegetal del suelo es un factor clave en la protección contra procesos erosivos, y es un componente básico de la agricultura de conservación debido a su capacidad de reducir drásticamente la tasa de erosión (Harden, 1988; Inbar \& Llerena, 2000). Por otra parte, las prácticas de labranza y control de arvenses típicamente utilizadas en cultivos de hortalizas restringen el mayor aprovechamiento de interacciones favorables entre el componente físico y biológico del suelo. Así, suelos cultivados con mayor frecuencia tienden a presentar comunidades de microorganismos menos estables y menos diversas que suelos cultivados con menor intensidad (Roger-Estrade et al., 2010). De forma similar, algunos estudios han reportado que el uso de coberturas como los acolchados vivos también favorecen el establecimiento de microecologías favorables en el suelo (Qian et al., 2015). No obstante, si bien la reducción de la labranza y una cobertura vegetal constante son aspectos deseables que reducen el riesgo de erosión del suelo, por otra parte, también pueden limitar el desarrollo fisiológico de los cultivos y, por ende, reducir sus rendimientos (Kołota \& Adamczewska-Sowińska, 2013). En general, las estrategias de labranza reducida y cobertura perenne del suelo como los acolchados vivos han sido implementadas con relativo éxito en cultivos extensivos como maíz, soya, trigo y frutales; sin embargo, en cultivos de hortalizas, los resultados de esta técnica han sido más variables y dependientes del cultivo y condiciones ambientales locales (Brandsæter et al., 1998).

Por lo expuesto anteriormente, realizar evaluaciones locales sobre el uso de acolchados vivos en cultivos hortícolas es clave para determinar la aplicabilidad de esta técnica de agricultura de conservación en zonas de producción específicas como la sierra ecuatoriana. En este trabajo reportamos una evaluación de dos tipos de acolchado vivo en dos cultivos representativos de la horticultura periurbana de Cuenca. El objetivo principal de este trabajo fue determinar los efectos que los acolchados vivos podrían tener sobre el desarrollo y rendimiento de cultivos hortícolas típicamente usados en rotación en esta zona.

\section{Materiales y Métodos}

\subsection{Ubicación y condiciones ambientales}

Los ensayos se realizaron en parcelas experimentales ubicadas en el campus Yanuncay de la Universidad de Cuenca durante el año 2018 (02 ${ }^{\circ}$ $55^{\prime} 15^{\prime} \mathrm{S}, 79^{\circ} 01^{\prime} 27^{\prime}$ 'W). Las condiciones ambientales en las que se desarrolló el experimento fueron representativas de aquellas observadas en zonas de producción periurbana alrededor de Cuenca, esto es, temperatura promedio de $13^{\circ} \mathrm{C}$ y pluviosidad bimodal con un promedio de precipitación anual acumulada de $780 \mathrm{~mm}$, que corresponde a un clima subtropical de montaña de acuerdo a la clasificación de Köppen. El suelo de las parcelas experimentales fue un vertisol ubicado en una terraza artificial y cultivado por al menos dos años previo al inicio del experimento dentro de una rotación de hortalizas.

\subsection{Condiciones experimentales y tratamientos}

Se desarrollaron dos ensayos consecutivos con lechuga y calabacín. La prueba con lechuga fue sembrada entre febrero y abril de 2018, usando trasplantes de 20 días de edad de la variedad Great Lakes 366 en camas de $0,8 \mathrm{~m}$ de ancho con tres hileras alternas con espaciamiento de $0,3 \mathrm{~m}$ entre hileras y 0,3 $\mathrm{m}$ entre plantas. El experimento con calabacín fue establecido entre septiembre y diciembre de 2018 usando trasplantes de 20 días de edad de la variedad 
Black Beauty en camas de $0.8 \mathrm{~m}$ de ancho con hileras dobles alternas y un espaciamiento de $0,6 \mathrm{~m}$ entre hileras y $0,6 \mathrm{~m}$ entre plantas. Ambos experimentos fueron desarrollados usando diseños de bloques completos al azar con cinco repeticiones. Cada bloque consistió en una cama de $0,8 \mathrm{~m}$ de ancho. Cada unidad experimental estuvo formada por una sección de cama de 2,9 $\mathrm{m}$ de largo (i.e. 0,8 $\mathrm{m}$ x 2,9 m) en el experimento con lechuga y de $4 \mathrm{~m}$ de largo (i.e. 0,8 m x $4 \mathrm{~m}$ ) en el experimento con calabacín. En el caso del ensayo con lechuga, la unidad de muestreo estuvo constituida por las 8 plantas centrales dentro de cada unidad experimental, mientras que en el caso del ensayo con calabacín estuvo compuesta por las 5 plantas centrales. En ambos cultivos se evaluaron los siguientes tres tratamientos: i) acolchado vivo de trébol rojo, ii) acolchado vivo de vegetación espontánea manejada y iii) un control sin acolchado.

El acolchado de trébol rojo fue sembrado un mes antes del trasplante justo después de la labranza y formación de las camas. Las semillas de trébol fueron sembradas al voleo a una tasa de $21,5 \mathrm{~g} / \mathrm{m}^{2}$ sobre el suelo irrigado. El acolchado de vegetación espontánea manejada consistió en toda la vegetación espontánea generada en la parcela experimental durante un mes después de la preparación del suelo como se indicó previamente. Antes del trasplante, ambos cultivos de acolchado fueron cortados a una altura de $\sim 5 \mathrm{~cm}$ desde el nivel del suelo, los residuos de este corte fueron esparcidos uniformemente sobre la unidad experimental. Las dos coberturas fueron revisadas semanalmente y el rebrote fue mantenido a $\sim 5 \mathrm{~cm}$ de altura con podas manuales por la duración del cultivo. El tratamiento control sin acolchado se mantuvo sin ningún tipo de vegetación extraña al cultivo durante todo el experimento a través de deshierbes manuales.

\subsection{Análisis de datos}

En ambos cultivos se registró la tasa de emisión foliar, el peso y tamaño del producto comercial y el rendimiento por planta. Además, se registró el $\mathrm{pH}$, conductividad eléctrica y densidad aparente del suelo antes y después del cada cultivo experimental. Los datos fueron analizados usando pruebas $t$ de Student para comparar estadísticamente las medias de cada uno de los tratamientos con acolchado utilizados contra el control.

\section{Resultados y discusión}

El efecto de la implementación de los acolchados en el crecimiento y rendimiento fue diferente para cada uno de los cultivos. En el caso de la lechuga, los dos tipos de acolchados redujeron la tasa de emisión foliar comparados contra el control; no obstante, en el caso del calabacín, sólo el acolchado de trébol redujo la tasa de emisión foliar (Figura 1). La tasa de emisión foliar fue utilizada como medida aproximada para evaluar el desarrollo del cultivo. Los resultados obtenidos indican que aquellos tratamientos en los que se observó menor tasa de emisión foliar con respecto al control presentaron retardo de crecimiento y consecuentemente sugieren la presencia de factores de estrés como inductores de esta respuesta. Reducciones en el crecimiento y rendimiento han sido reportados como los principales limitantes de la implementación de los acolchados vivos en cultivos hortícolas (Kołota et al., 2013; Masiunas, 1998). Otros estudios muestran que las posibles causas de esta reducción de crecimiento, ha sido la competencia por recursos como nutrientes, agua y luz entre el acolchado vivo y el cultivo principal, además de la liberación de aleloquímicos (Kołota et al., 2013; Masiunas, 1998).

No obstante, en la presente investigación, la reducción del crecimiento no siempre estuvo asociada a una disminución del rendimiento. Por ejemplo, en el caso del cultivo de lechuga, los tres tratamientos alcanzaron a generar el mismo número de hojas hacia el final del ciclo y produjeron repollos de tamaño y peso similar (Tabla 1), mostrando que, si bien el crecimiento se vio retardado, este retraso no afectó al rendimiento. En contraste, en el cultivo de calabacín la reducción de la tasa de emisión foliar en el cultivo con acolchado de trébol sí coincidió con una reducción estadísticamente significativa del rendimiento por planta, derivada de una reducción en el número, peso y tamaño (largo) de los frutos (Tabla 2). Existen estudios en que los acolchados mejoraron la productividad o biomasa de las plantas (Li et al., 2018; Lal, 1997), en donde los acolchados de paja aumentaron el rendimiento de cultivos de ciclo mediano como la papa en un $16,0 \%$ por efecto de retención del agua y además redujeron la erosión del suelo. En cambio, en otros agroecosistemas, se han reportado efectos negativos de los acolchados de trébol en cultivos como col o calabaza similar a los observados en el 
actual estudio (Brandsæter et al., 1998; Galloway \& Weston, 1996; Masiunas, 1998). No obstante, en dichas investigaciones el acolchado de trébol fue aplicado como una mezcla con otros acolchados vivos como el centeno; el principal problema derivado de su aplicación fue que el trébol logró sobrepasar al cultivo principal en su crecimiento. En nuestro caso, la altura del cultivo de acolchado fue mantenido a través de cortes a una altura de $5 \mathrm{~cm}$, por lo que, la competencia por nutrientes o agua podrían estar más relacionada a la reducción de rendimiento observada en el ensayo con calabacín.
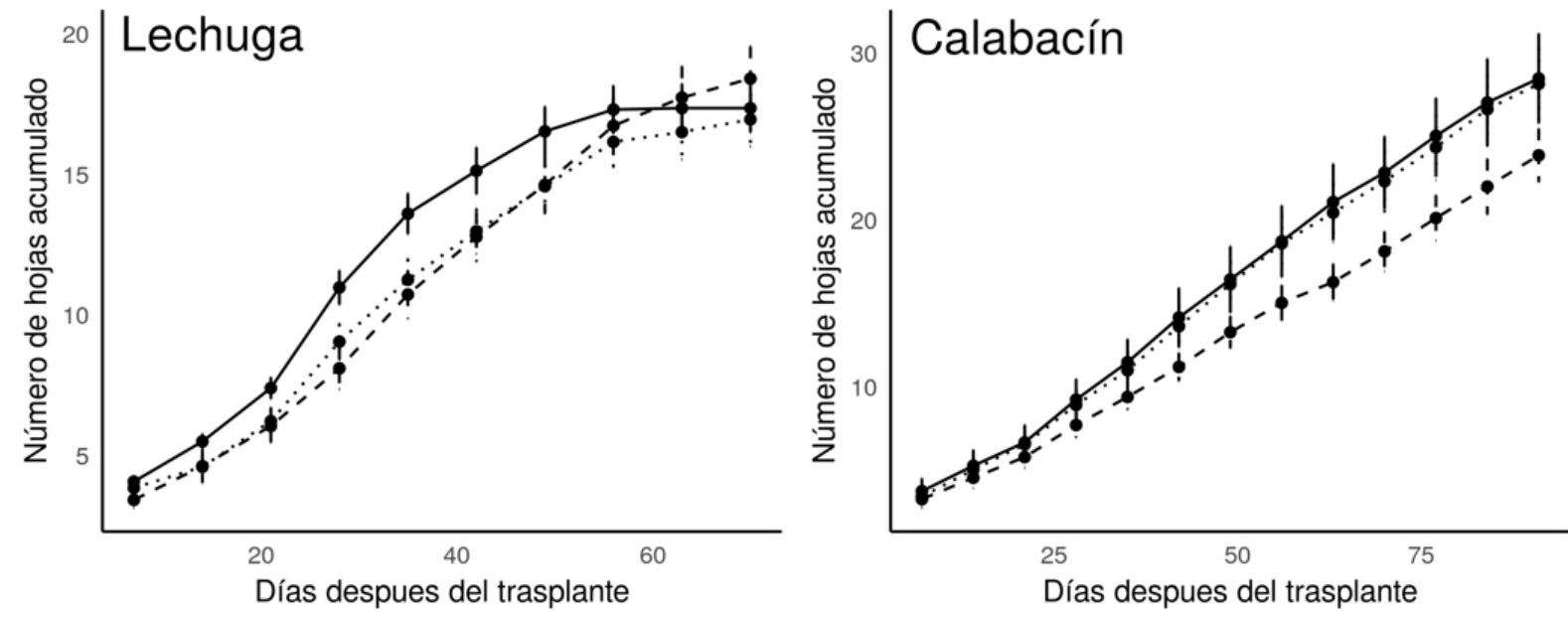

Figura 1. Número de hojas acumuladas en cultivos de lechuga y calabacín bajo dos sistemas de acolchado vivo: trébol (línea de rayas), vegetación espontánea manejada (línea de puntos) y control sin acolchado (línea sólida). Cada punto representa la media \pm el error estándar $(n=5)$.

Tabla 1. Rendimiento, peso y tamaño de repollos de lechuga cultivados bajo dos sistemas de acolchado vivo. Los valores son medias \pm error estándar $(\mathrm{n}=5)$. Asteriscos indican diferencias estadísticamente significativas entre el tratamiento y el control sin cobertura (valor $\mathrm{p}<0.05$, prueba $\mathrm{t})$

\begin{tabular}{lccccc}
\hline & $\begin{array}{c}\text { Rendimiento }(\mathrm{Kg} / \\
\text { planta })\end{array}$ & Frutos por planta & Peso del fruto $(\mathrm{g})$ & $\begin{array}{c}\text { Largo del fruto } \\
(\mathrm{cm})\end{array}$ & $\begin{array}{c}\text { Diámetro del fruto } \\
(\mathrm{cm})\end{array}$ \\
\hline Trébol & $1,35 \pm 0,31^{*}$ & $1,24 \pm 0,19^{*}$ & $209,08 \pm 23,34^{*}$ & $17,61 \pm 0,86^{*}$ & $4,54 \pm 0,14$ \\
$\begin{array}{l}\text { Vegetación espontánea } \\
\text { manejada }\end{array}$ & $3,44 \pm 1,11$ & $2,32 \pm 0,19$ & $258,11 \pm 45,20$ & $19,80 \pm 1,54$ & $4,61 \pm 0,31$ \\
Control sin acolchado & $4,43 \pm 0,7$ & $2,8 \pm 0,34$ & $304,49 \pm 23,60$ & $20,48 \pm 0,43$ & $4,95 \pm 0,16$ \\
\hline
\end{tabular}

Tabla 2. Rendimiento, peso y tamaño de frutos de calabacín cultivados bajo dos sistemas de acolchado vivo. Los valores son medias \pm error estándar $(\mathrm{n}=5)$. Asteriscos indican diferencias estadísticamente significativas entre el tratamiento y el control sin cobertura (valor $\mathrm{p}<0.05$, prueba $\mathrm{t})$.

Rendimiento (g/planta)

Altura del repollo (cm)

Diámetro del repollo $(\mathrm{cm})$

\begin{tabular}{|c|c|c|c|}
\hline Trébol & $509,02 \pm 92,84$ & $10,46 \pm 0,66$ & $9,2 \pm 0,84$ \\
\hline Vegetación espontánea manejada & $423,62 \pm 68,52$ & $12,40 \pm 0,66$ & $10,12 \pm 0,77$ \\
\hline Control sin acolchado & $539,38 \pm 60,04$ & $12,72 \pm 0,86$ & $10,24 \pm 0,24$ \\
\hline
\end{tabular}


Además de las variables de cultivo, en este estudio registramos también un pequeño panel de variables edáficas, específicamente densidad aparente, $\mathrm{pH}$ y conductividad eléctrica. Los cambios en las propiedades físico-químicas del suelo derivados de la implementación de cultivos de acolchado han sido reportados como favorables en términos generales (Kołota \& Adamczewska-Sowińska, 2013). Entre los principales efectos beneficiosos de la implementación de acolchados vivos están la reducción de procesos de compactación de suelo y una mejor estabilidad de los agregados. En nuestro ensayo, el acolchado de vegetación espontánea manejada registró consistentemente los más bajos valores de densidad aparente de los que se puede inferir una menor compactación. En el ensayo con lechuga, tanto el cultivo acolchado con trébol como el control sin cobertura mostraron una compactación normal producto de la acción de los elementos hacia el final del ciclo de producción, mientras que el cultivo con acolchado de vegetación espontánea manejada mantuvo el mismo valor de densidad aparente que el suelo recién preparado (Tabla 3). En el caso del cultivo de calabacín, los resultados fueron un poco más variables; no obstante, el acolchado con vegetación espontánea registró los valores más bajos de densidad aparente. Una posible explicación a esta diferencia entre los resultados de ambos ensayos puede derivarse de que el ensayo con lechuga se desarrolló durante la temporada lluviosa, mientras que el ensayo con calabacín se realizó en una época con menos precipitaciones. En el caso del pH, en todos los casos se registró una reducción entre los valores iniciales y finales en ambos ensayos; no obstante, debido a la variabilidad inicial de los valores de $\mathrm{pH}$ no fue posible distinguir efectos claros derivados del tipo de acolchado utilizado. En el caso de la conductividad eléctrica, las respuestas fueron consistentes entre los tres tratamientos en ambos ensayos, sin embargo, los valores de conductividad eléctrica fueron mayores al final del ensayo con lechuga y menores al final del ensayo con calabacín respecto a los valores de conductividad al inicio de cada ensayo. Esta diferencia en el sentido del cambio entre valores iniciales y finales podría deberse a particularidades de cada cultivo, condiciones ambientales durante el desarrollo del ensayo $u$ otros factores no considerados en el planteamiento experimental.

Tabla 3. Densidad aparente, $\mathrm{pH}$ y conductividad eléctrica del suelo al inicio y final del ciclo de producción de lechuga y calabacín cultivados bajo dos sistemas de acolchado vivo

\begin{tabular}{|c|c|c|c|c|c|c|}
\hline & \multicolumn{2}{|c|}{$\begin{array}{c}\text { Densidad aparente } \\
\left(\mathrm{g} / \mathrm{cm}^{3}\right)\end{array}$} & \multicolumn{2}{|c|}{$\mathrm{pH}$} & \multicolumn{2}{|c|}{$\begin{array}{c}\mathrm{CE} \\
(\mathrm{mS} / \mathrm{m})\end{array}$} \\
\hline & Inicio & Final & Inicio & Final & Inicio & Final \\
\hline \multicolumn{7}{|l|}{ Lechuga } \\
\hline Trébol & $1,15 \pm 0,03$ & $1,26 \pm 0,03$ & $7,42 \pm 0,06$ & $7,08 \pm 0,03$ & $144 \pm 5$ & $304 \pm 15$ \\
\hline $\begin{array}{l}\text { Vegetación } \\
\text { espontánea } \\
\text { manejada }\end{array}$ & $1,15 \pm 0,01$ & $1,16 \pm 0,03$ & $7,51 \pm 0,06$ & $7,18 \pm 0,03$ & $131 \pm 3$ & $247 \pm 23$ \\
\hline $\begin{array}{l}\text { Control sin } \\
\text { acolchado }\end{array}$ & $1,13 \pm 0,01$ & $1,23 \pm 0,02$ & $7,38 \pm 0,06$ & $7,16 \pm 0,05$ & $138 \pm 2$ & $231 \pm 4$ \\
\hline \multicolumn{7}{|l|}{ Calabacín } \\
\hline Trébol & $1,29 \pm 0,03$ & $1,29 \pm 0,12$ & $7,33 \pm 0,04$ & $7,10 \pm 0,03$ & $222 \pm 14$ & $136 \pm 8$ \\
\hline $\begin{array}{l}\text { Vegetación } \\
\text { espontánea } \\
\text { manejada }\end{array}$ & $1,33 \pm 0,04$ & $1,14 \pm 0,06$ & $7,17 \pm 0,13$ & $7,10 \pm 0,05$ & $202 \pm 7$ & $122 \pm 7$ \\
\hline $\begin{array}{l}\text { Control sin } \\
\text { acolchado }\end{array}$ & $1,33 \pm 0,06$ & $1,19 \pm 0,06$ & $7,22 \pm 0,06$ & $7,06 \pm 0,07$ & $234 \pm 15$ & $124 \pm 11$ \\
\hline
\end{tabular}

En conjunto, los resultados obtenidos indican que los cultivos desarrollados sobre acolchados vivos lograron producir rendimientos equivalentes a los observados en los controles sin cobertura. El tipo de acolchado que mostró resultados más consistentes fue el acolchado con vegetación espontánea 
manejada, en el cual se registraron rendimientos similares al control sin cobertura en ambos cultivos. No obstante, efectos negativos en el crecimiento y rendimiento fueron también detectados sugiriendo que los acolchados evaluados aquí deben ser optimizados. De forma similar, es posible que la implementación de esta práctica incremente costos de producción derivados de un mayor requerimiento de trabajo para mantener los cultivos de acolchado a alturas no competitivas con el cultivo principal. En este estudio, nos centramos en describir, de forma inicial, la respuesta del cultivo y en menor medida algunas variables edáficas, no obstante, existen otras variables que podrían mejorar futuras evaluaciones de la viabilidad de uso de esta técnica en sistemas de producción de hortalizas en el Ecuador. Por ejemplo, otros autores han manifestado efectos beneficiosos de los acolchados vivos en la reducción de ataques de plagas lepidópteras e incremento de la diversidad de potenciales enemigos naturales de plagas en brócoli (Hooks \& Johnson, 2004). De forma similar, se han reportado reducciones de los niveles de daños derivados de ataques de áfidos y trips en brasicáceas y puerros (Costello \& Altieri, 1994; Theunissen \& Schelling, 1998), así como reducciones en necrosis foliar asociada a enfermedades (Abdul-Baki et al., 1996). Por otra parte, también se ha informado un incremento de la actividad y presencia de organismos benéficos del suelo como lombrices y hongos micorrízicos arbusculares en suelos manejados con cultivos de acolchado (Deguchi et al., 2007; Hartwig \& Ammon, 2002; Pelosi, Bertrand, \& Roger-Estrade, 2009). Existen reportes donde los acolchados o vegetación en zonas de pendiente han mejorado la evaporación y la capacidad del suelo para mantener

\section{Referencias}

Abdul-Baki, A. A., Stommel, J. R., Watada, A. E., Teasdale, J. R., \& Morse, R. D. (1996). Hairy Vetch Mulch Favorably Impacts Yield of Processing Tomatoes. HortScience, 31(3), 338-340.https://doi.org/10.21273/HORTSCI.31.3.338

Blanco-Canqui, H., \& Lal, R. (2009). Crop residue removal impacts on soil productivity and environmental quality. Critical reviews una misma temperatura, así como preservar macro y micronutrientes (Lal, 1998; Blanco-Canqui \& Lal, 2009). En el Ecuador, existen muy pocos trabajos relacionados con el uso de acolchados vivos en cultivos hortícolas, en este contexto, los resultados alcanzados en la presente investigación ofrecen una evaluación inicial de la viabilidad en el uso de esta práctica como estrategia para mejorar la sostenibilidad de los sistemas de producción hortícolas a través de su intensificación sostenible.

\section{Conclusiones}

Los rendimientos de lechuga y calabacín cultivados sobre acolchado vivo de vegetación espontánea fueron similares a los observados en los respectivos cultivos de control sin cobertura. En el caso del acolchado con trébol, este produjo rendimientos similares en el cultivo de lechuga, pero redujo significativamente el rendimiento de calabacín. En conjunto estos resultados sugieren que esta práctica podría ser aplicable en el contexto de la horticultura periurbana de Cuenca; no obstante, se identifican importantes limitaciones que deben ser resueltas antes de poder ser recomendada en forma general.

\section{Agradecimientos}

Este trabajo fue financiado por fondos competitivos de investigación de la Universidad de Cuenca (XIV Concurso Universitario de Proyectos de Investigación) a través del proyecto "Evaluación integral de los sistemas de producción hortícola periurbanos de Cuenca y actitud de los productores sobre la adoptabilidad de estrategias de intensificación sustentable".

in plant science, 28(3), 139-163. https://doi. org/10.1080/07352680902776507

Brandsæter, L. O., Netland, J., \& Meadow, R. (1998). Yields, Weeds, Pests and Soil Nitrogen in a White Cabbage-Living Mulch System. Biological Agriculture \& Horticulture, 16(3), 291-309. https://doi.org/10.1080/01448765.19 98.10823201 
Costello, M., \& Altieri, M. (1994). Livina mulches suppress aphids in broccoli. California Agriculture, 48(4), 24-28.

Deguchi, S., Shimazaki, Y., Uozumi, S., Tawaraya, K., Kawamoto, H., \& Tanaka, O. (2007). White clover living mulch increases the yield of silage corn via arbuscular mycorrhizal fungus colonization. Plant and Soil, 291(1), 291-299. https:// doi.org/10.1007/s11104-007-9194-8

FAO. (2005). Final Report of the Project "Global Inventory, Reference Materials and Food, Safety Training Programme for Improving the Quality and safety of Fresh Fruits and Vegetables". Obtenido de: http://www.fao.org/ag/agn/ pfl_report_en/it.html

Galloway, B. A., \& Weston, L. A. (1996). Influence of Cover Crop and Herbicide Treatment on Weed Control and Yield in No-Till Sweet Corn (Zea mays L.) and Pumpkin (Cucurbita maxima Duch). Weed Technology, 10(2), 341-346.

Harden, C. (1988). Mesoscale Estimation of Soil Erosion in the Rio Ambato Drainage, Ecuadorian Sierra. Mountain Research and Development, 8(4), 331-341. https://doi.org/10.2307/3673556

Hartwig, N. L., \& Ammon, H. U. (2002). Cover Crops and Living Mulches. Weed Science, 50(6), 688-699. Recuperado de JSTOR.

Hooks, C. R., \& Johnson, M. W. (2004). Using undersown clovers as living mulches: Effects on yields, lepidopterous pest infestations, and spider densities in a Hawaiian broccoli agroecosystem. International Journal of Pest Management, 50(2), 115-120. https://doi.org/10.1 080/09670870410001663462

Inbar, M., \& Llerena, C. A. (2000). Erosion Processes in High Mountain Agricultural Terraces in Peru. Mountain Research and Development, 20(1), 72-79. https://doi.org/10.1659/0276-474 1(2000)020[0072:EPIHMA]2.0.CO;2

Lal, R. (1997). Mulching effects on runoff, soil erosion, and crop response on alfisols in Western Nigeria. Journal of sustainable agriculture,
11(2-3), 135-154. https://doi.org/10.1300/ J064v11n02_10

Lal, R. (1998). Soil erosion impact on agronomic productivity and environment quality. Critical reviews in plant sciences, 17(4), 319-464. https://doi.org/10.1080/07352689891304249

Li, Q., Li, H., Zhang, L., Zhang, S., \& Chen, Y. (2018). Mulching improves yield and water-use efficiency of potato cropping in China: A meta-analysis. Field crops research, 221, 50-60.

Kołota, E., \& Adamczewska-Sowińska, K. (2013). Living Mulches in vegetable crops production: Perspectives and limitations (A review). Acta Scientiarum Polonorum-Hortorum Cultus, 12(6), 127-142.

Masiunas, J. B. (1998). Production of Vegetables Using Cover Crop and Living Mulches-A Review. Journal of Vegetable Crop Production, 4(1), 1131. https://doi.org/10.1300/J068v04n01_03

Pelosi, C., Bertrand, M., \& Roger-Estrade, J. (2009). Earthworm community in conventional, organic and direct seeding with living mulch cropping systems. Agronomy for Sustainable Development, 29(2), 287-295. https://oi. org/10.1051/agro/2008069

Qian, X., Gu, J., Pan, H., Zhang, K., Sun, W., Wang, X., \& Gao, H. (2015). Effects of living mulches on the soil nutrient contents, enzyme activities, and bacterial community diversities of apple orchard soils. European Journal of Soil Biology, 70, 23-30. https://doi.org/10.1016/j. ejsobi.2015.06.005

Roger-Estrade, J., Anger, C., Bertrand, M., \& Richard, G. (2010). Tillage and soil ecology: Partners for sustainable agriculture. Soil and Tillage Research, 111(1), 33-40. https://doi.or$\mathrm{g} / 10.1016 / \mathrm{j}$. still.2010.08.010

Theunissen, J., \& Schelling, G. (1998). Infestation of leek by Thrips tabaci as related to spatial and temporal patterns of undersowing. BioControl, 43(1), 107-119. https://doi.org/10.1023/A:1009986917903 